\section{SOSTENIBILIDAD Y TURISMO EN LOS PAISAJES CULTURALES DE LA INDUSTRIALIZACIÓN}

\author{
Carlos J. Pardo Abad \\ Universidad Nacional de Educación a Distancia \\ ORCID iD: http://orcid.org/0000 000300475834 \\ cjpardo@geo.uned.es
}

Cómo citar este artículo/Citation: Pardo Abad, C. J. (2017). Sostenibilidad y turismo en los paisajes culturales de la industrialización. Arbor, 193 (785): a400. doi: http://dx.doi. org/10.3989/arbor.2017.785n3006

Recibido: 10 noviembre 2016. Aceptado: 6 junio 2017

RESUMEN: Las antiguas zonas mineras e industriales, muchas de ellas ya abandonadas tras una prolongada actividad extractiva y productiva, albergan frecuentemente buenas experiencias de recuperación del patrimonio asociado y sus paisajes. Impulsados inicialmente los proyectos desde las administraciones públicas, sobre todo de ámbito municipal, con participación activa de las empresas propietarias y la comunidad local, hoy son un extraordinario referente de puesta en valor de un legado muy peculiar que ofrece amplias posibilidades de reutilización bajo la perspectiva irrenunciable del desarrollo sostenible.

PALABRAS CLAVE: Patrimonio industrial; turismo; proyectos de recuperación; desarrollo sostenible; ecomuseos.
SUSTAINABILITY AND TOURISM

IN THE CULTURAL LANDSCAPES OF INDUSTRIALIZATION

Copyright: (C) 2017 CSIC. Este es un artículo de acceso abierto distribuido bajo los términos de la licencia Creative Commons Attribution (CC BY) España 3.0.

ABSTRACT: Old mining and industrial areas, many of them already abandoned after prolonged extractive and productive activity, often put up good experiences associated with recovery heritage and landscapes. Initially promoted by public administrations, particularly municipal bodies, with the active participation of private companies and the local community, nowadays these projects are an extraordinary reference for valuing a peculiar legacy that offers extensive possibilities for reuse under the indispensable perspective of sustainable development.

KEYWORDS: Industrial heritage; tourism; recovery projects; sustainable development; ecomuseums. 


\section{INTRODUCCIÓN}

Los viajes ilustrados del siglo XVIII y el interés que se despertó entonces hacia las más variadas observaciones naturales y culturales, entre las que se encontraba el progreso técnico vinculado a la producción, impulsaron la atracción y el acercamiento intelectual hacia las fábricas a lo largo del siglo XIX. La fascinación decayó con la llegada del siglo XX. Fue entonces cuando lo cultural empezó a identificarse con lo artístico y el arte se convirtió en el gran protagonista de lo que ya se podría denominar como turismo. La intensificación industrial que caracterizó a las primeras décadas del siglo XX convirtió sus avances y manifestaciones materiales en algo habitual y asumido, con menor poder de seducción. El turismo de masas de base heliotrópica que se inició tras la Segunda Guerra Mundial acabó por liquidar el interés hacia las industrias y la industrialización.

Ese interés volvió a despertarse con la crisis económica de mediados de la década de 1970, considerándose el final precipitado de un periodo que había logrado marcar profundamente las identidades básicas de la sociedad industrial. Muchas voces reivindicaron la conservación de los restos productivos más significativos, que permitiesen mantener vivo el recuerdo de un pasado aún no muy lejano. Fue un proceso lento, pero progresivo, de patrimonialización de lo industrial en el que jugaron un papel destacado las emociones y los recuerdos individuales y colectivos. Las propuestas de conservación e introducción de nuevas funciones en los espacios más singulares comenzaron a materializarse con proyectos de muy distinta naturaleza y con intensidades diferenciadas según regiones y países.

Las antiguas fábricas y áreas mineras recuperadas se acabaron convirtiendo en una de las facetas más visibles de las nuevas tendencias culturales. Este turismo se aleja de la densificación y homogeneización de la oferta en otros turismos, creando productos nuevos y competitivos en el marco de una diversificación cada vez más compleja, lo que obliga a aplicar proyectos de intervención que consideren siempre el entramado territorial, la participación de la población local y una gestión basada en principios racionales y sostenibles. Por otro lado, se trata de un turismo en un contexto de cambio social y de nuevo modelo productivo en el marco de la globalización (Zárate Martín, 2011). Solo así se garantiza el éxito de los destinos que estuvieron vinculados a la producción.

\section{ESTADO DE LA CUESTIÓN Y PERSPECTIVAS TEÓRICAS}

El interés por el patrimonio industrial en España surge en la década de 1980 . Es más tardío que en otros países europeos, pero con el mismo poder de atracción: más de 100.000 visitas anuales en el Museo de la Ciencia y de la Técnica de Tarrasa en 2016, 117.000 en el Museo del Ferrocarril de Madrid y 89.235 en el Parque Minero de Riotinto, también en 2016. De algún modo, se ha devuelto a la gente un patrimonio que fue de la gente (Samuel, 1994). Pero además, se ha pasado de la puesta en valor del elemento construido a la necesidad de tratarlo de manera integrada con el paisaje y el territorio en los que se ubica. Se asume, por lo tanto, que es necesario adoptar una visión más amplia en la que se tengan en cuenta aspectos sociales, económicos y culturales, que acaban por reafirmar la identidad colectiva y promover una opinión favorable a la conservación y nuevo uso (Casanelles, 1998; Feliú Torras, 1998; Hidalgo, 2010).

El crecimiento del turismo cultural como fenómeno de masas, iniciado a finales del XX, favoreció la valoración del concepto de patrimonio como un legado no vinculado tanto a la estética como a la amplia herencia transmitida por una comunidad en un espacio determinado (Sabaté Bel, 2004). Fue un cambio significativo que resultó de la propia evolución de las ideas en el campo de la protección de los componentes naturales y culturales y del concepto de paisaje cultural. Aunque los orígenes del término paisaje cultural se pueden encontrar en algunos geógrafos franceses y alemanes de finales del siglo XIX (Ratzel, (1844-1904), Schlütter (1872-1959), Vidal de la Blache (1845-1918), la acepción actual aparece a principios del siglo XX con la Escuela de Berkeley y especialmente con la figura de Carl Sauer (1889-1975), que lo define como la acción de un grupo social sobre un paisaje natural.

El legado de esos autores se retoma en el último tercio del siglo XX, de manera indirecta por la UNESCO en 1972, con la Convención del Patrimonio Mundial Cultural y Natural, y de modo explícito en 1992 por las Directrices de la UNESCO para la aplicación de la anterior Convención, definiendo por primera vez en su Artículo 1 el concepto de paisaje cultural: "los paisajes culturales son bienes culturales y representan las "obras conjuntas del hombre y la naturaleza»". Otras referencias obligadas son la Convención del Paisaje Europeo de Florencia de 2000 y, desde un punto de vista operativo, las actuaciones de conservación del National Park Service de Estados Unidos, la creación del Parque Cultural del Carbón en 1972, un año antes de la mítica recuperación de New Lanark en Escocia y de que en 1975 se creara el New Lanark Conservation Trust. La importancia de este paisaje fue reconocida por la UNESCO, declarándolo Patrimonio de la Huma- 
nidad en 2001 (www.whc.unesco.org/es/list), y en 1978 se protegió como Patrimonio de la Humanidad la mina de sal de Wieliczka, próxima a Cracovia (Polonia).

El estudio científico del patrimonio industrial adquirió temprano desarrollo en Inglaterra, Francia y Alemania; primero en torno a los ejemplos más significativos de las industrias del siglo XIX, para extenderse después a otros casos menos grandiosos o singulares y a la interpretación global de los paisajes de la industrialización, promoviendo sus valores culturales, la preservación de su patrimonio histórico y la recuperación recreativa (Cossons, 2012; Albrecht, 2012). Las actividades industriales y mineras pasaron a ser consideradas por su capacidad configuradora de paisajes culturales, recuperándose enclaves tan emblemáticos como Lowell, Blackstone y Lackawana en forma de parques patrimoniales. Este nuevo concepto integral quedó posteriormente recogido en la Convención Europea del Paisaje, firmada en Florencia en el año 2000. Unos años antes, los paisajes culturales fueron reconocidos oficialmente como parte integrante de la Lista de Patrimonio Mundial a partir de 1992, incluyéndose también los industriales. En este contexto general se enmarca la aprobación en España de un Plan Nacional de Patrimonio Industrial, que comenzó en el año 2001 y en 2002 se elaboró un catálogo inicial de bienes de intervención prioritaria. Más recientemente, en 2012, se aprobó un Plan Nacional de Paisajes Culturales, en donde se contemplan como categoría específica los paisajes heredados de la industrialización.

Por otra parte, en las dos últimas décadas han proliferado los debates sobre la recuperación e introducción de nuevas actividades en los antiguos espacios industriales. Son muchos los que defienden que este turismo es compatible con el desarrollo sostenible (Sharpley, 2000; Cole, 2004), destacando como aspecto más relevante sus beneficios de tipo social, económico y ambiental. Sin embargo, también se insiste en las dificultades que existen para la generación de riqueza y empleo en muchos de los paisajes industriales, sobre todo cuando se ubican en ámbitos rurales, por la complejidad que resulta de la intervención de actores de muy distinta naturaleza y la presencia de intereses dispares y contrapuestos (Valenzuela, Palacios e Hidalgo, 2008).

La creciente sensibilización de las instancias oficiales y de la sociedad hacia la preservación ha logrado alcanzar a la noción de paisaje industrial, considerándolo como recurso no renovable y objeto de protección (Vahí, 2010; Castillo, López y Millán, 2010; Benito y Alonso, 2012). Esto ha sido fundamental para la con- sideración del patrimonio industrial como elemento de identidad y cohesión de las comunidades locales (Benito y Alonso, 2012) y como base para nuevos usos sostenibles (Pardo Abad, 2016).

El concepto de sostenibilidad arranca de la década de 1970, con la celebración de algunas conferencias (Estocolmo, 1972) y la firma de algunos convenios (París, 1972) que representan los primeros compromisos internacionales para la conservación de la naturaleza y la cultura (Convención del Patrimonio Mundial Cultural y Natural de la UNESCO de 1972). Después vino la redacción de informes (Informe Brundtland, 1987), la celebración de reuniones internacionales (Cumbre de la Tierra o de Río de 1992; Cumbre Mundial sobre Desarrollo Sostenible, 2002; Cumbre de Naciones Unidas Río+ 20, en junio de 2012) o acuerdos sectoriales concretos (Carta Europea de Turismo Sostenible, 2007), en los que se destaca que las motivaciones culturales son cada vez más importantes en los desplazamientos turísticos y la calidad de los destinos pasa por el uso sostenible del patrimonio ${ }^{1}$.

Algunos autores apuntan que el concepto de la sostenibilidad se ha aplicado al turismo con mucha ambigüedad, agravado por la gran profusión de formas específicas del turismo y de definiciones poco precisas de desarrollo sostenible (Butler, 1999). Lo más extendido ha sido reivindicar que cualquier turismo de pequeña escala es sostenible, focalizado en lo ambiental y cultural y con participación de la comunidad local, pero incluso ahí consideramos absolutamente necesario ahondar más en la conceptualización de la sostenibilidad y en el empleo de indicadores que permitan analizar la situación real y los efectos de futuro. Se ha sugerido también que es imprescindible prestar atención en este tema a la perspectiva económica, ecológica y ambiental (tanto a nivel físico como humano) y de viabilidad de los proyectos turísticos a largo plazo (Coccossis, 1996). En otros casos, el turismo sostenible ha sido entendido solo como forma duradera de turismo, en lugar de referirlo a la viabilidad de la actividad sin degradación ambiental.

Algunas aportaciones destacan los vínculos del patrimonio industrial con el turismo sostenible, enfatizando los beneficios de la recuperación de paisajes y construcciones abandonadas (Jansen-Verbeke, 1999; Butler, 1999; Sabaté Bel, 2004; Álvarez, 2010; Pardo Abad, 2014). La reutilización de edificaciones, la mejora ambiental del territorio, el uso turístico racional y la creación de proyectos y empresas de base local se enmarcan en los planteamientos de la sostenibilidad. En la búsqueda de actividades alternativas, el patri- 
monio industrial se ha convertido en una oportunidad real para relanzar las diferentes opciones de la reconversión turística (Edwards y Llurdés, 1996; Jones y Munday, 2001; Hospers, 2002; Dambron, 2004; Pérez y Parra, 2004; Pardo Abad, 2010; Benito, 2012).

La geografía ha necesitado bastante tiempo para considerar el patrimonio industrial como un elemento básico de los paisajes y de la cultura territorial. En el marco más reciente de la geografía postmoderna, fuertemente influida por la fenomenología y el existencialismo, que considera los distintos espacios como productos sociales singulares e irrepetibles, el legado industrial y su uso turístico se vinculan con la preservación de las señas de identidad de las comunidades locales. Y esta consideración, nacida de una nueva geografía, o de una geografía reconstituida, ha dado pie a la reivindicación de lo local frente a lo global, pero no como contraposición sino a través de las interrelaciones entre ambas escalas espaciales. Esto llevó a Benko y Strohmayer (1997) a acuñar el término de glocal. Bajo esa perspectiva, la geografía se interesa por el análisis de los lugares concretos, de límites imprecisos y confluencia de identidades y unicidades que rompen el nivel "material" y reconocido de las regiones estudiadas por la geografía clásica.

\section{PATRIMONIO INDUSTRIAL Y TURISMO SOSTENIBLE}

El paisaje de las áreas industriales es muy singular y producto de la concentración de fábricas, instalaciones mineras, almacenes, infraestructuras ferroviarias, etc., es decir, un complejo entramado que exige un tratamiento específico de los restos tras el cierre y abandono. El reconocimiento a su valor, sobre todo en relación con la primera industrialización, originó una corriente interesada por el paisaje fabril en las primeras décadas del siglo XX, así como por los artefactos industriales del pasado y las posibilidades para la recuperación de los grandes contenedores productivos (Stuart, 2012). En 1939 se abrió un primer museo de la industria del algodón en Styal (Cheshire) y en 1934 se incluyó en el catálogo de bienes inmuebles protegidos (Listed Buildings) el Ironbridge, puente de hierro de $1779^{2}$ que cruza el río Severn, en Shropshire, Inglaterra. A partir de 1970, la preocupación por la conservación industrial y su puesta en valor se generalizó, coincidiendo con la crisis del petróleo, la desindustrialización, la reconversión de los medios de producción y el urbanismo de la austeridad.

En las ciudades, esta concepción territorial y paisajista centró algunos trabajos sobre el cierre de viejas fábricas y la aparición de los denominados vacíos industriales (Pardo Abad, 1990), claros síntomas de la crisis metropolitana experimentada en la segunda mitad del siglo XX bajo los efectos del cambio de modelo productivo y urbano inducido por la globalización económica y la acelerada revolución tecnológica. Con el tiempo se pudo comprobar la capacidad de respuesta y la variedad de soluciones aportadas, todo ello en un nuevo contexto económico y urbanístico que propiciaba la recuperación de los espacios interiores de la ciudad (Zárate Martín, 2012).

En cuanto a la recuperación del patrimonio industrial y la puesta en valor de los paisajes asociados hay que tener en cuenta que son fenómenos relacionados con la sensación evocadora y nostálgica que causa la herencia de la revolución industrial. Y quizá con más fuerza que en otros legados, debido al cúmulo de sentimientos colectivos que aún perviven en los territorios de tradición industrial. Enlazando con los conceptos de 'espacio vivido' y de 'lugar', como centros de significado y focos de vinculaciones emocionales, existen muchos valores añadidos en este legado que dan vigor y dinamismo a su revalorización.

La Carta Mundial del Turismo Sostenible fue adoptada en 2015 en la Cumbre Mundial de Turismo Sostenible. De carácter generalista, y reiterando la validez actual de la Carta Mundial de Turismo Sostenible del año 1995, insiste en la necesidad de orientar el turismo hacia vías integradoras y sostenibles. En la Carta se hace especial hincapié en que la actividad turística debe convertirse en impulsora de las economías locales y contribuir a la participación activa de las comunidades locales como parte inexcusable en la gestión responsable de los destinos turísticos.

Aun sin realizar ninguna referencia explícita a un tipo concreto de turismo, el documento insiste permanentemente en la idea de que esta actividad debe reforzar el papel decisivo del patrimonio material e inmaterial. Siguiendo esta línea argumental, el patrimonio industrial puede considerarse como un elemento más que contribuye a alcanzar ese objetivo general, consolidando la identidad y la diversidad culturales como puntos de referencia imprescindibles para el desarrollo de unos destinos específicos, pero conformadores también de los activos turísticos más generales y de conjunto.

Los problemas de conservación del patrimonio industrial han de encontrar en la actividad turística el modo de solución por los efectos inducidos sobre la economía y cultura locales, la cooperación entre agentes y organismos públicos y privados, la innova- 
ción en la oferta y sobre la responsabilidad compartida en la preservación de los bienes industriales heredados. Todo ello sin olvidar la atención que se ha de prestar al frágil patrimonio cultural inmaterial de los destinos. De especial relevancia en las zonas de antigua industrialización, se ha transmitido de generación en generación y ha creado en los grupos locales un sentimiento de identidad y continuidad. El uso responsable de este patrimonio vivo con fines turísticos puede generar nuevas oportunidades de desarrollo, pero evitando dos tendencias bastante extendidas en la actualidad: la degradación y la homogeneización. Es una forma más de ahondar en la sostenibilidad turística, conservando la autenticidad y la integridad de los lugares y destinos de patrimonio industrial (figura 1).

Figura 1. Obras de recuperación en el Parque Minero de Almadén (Ciudad Real)

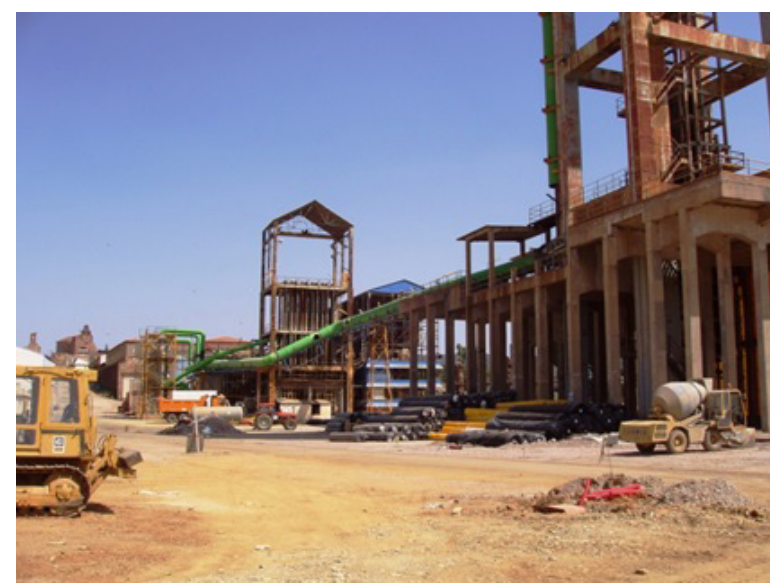

Fotografía tomada en agosto de 2007.

El Plan Nacional de Patrimonio Industrial, aprobado inicialmente en 2001 y revisado en el año 2011, enfatiza también la necesidad de la sostenibilidad. Es, de algún modo, un cambio de paradigma, un nuevo enfoque con gran repercusión en el patrimonio industrial por lo que representa de conservación preventiva de los bienes, la implicación de la sociedad y sus instituciones, la gestión proactiva de los recursos y el uso responsable por los ciudadanos. Estas son las garantías de que el legado material e inmaterial de la revolución industrial permanezca como bien social, factor de cohesión y testigo de la historia de los territorios. El patrimonio industrial, así como sus extraordinarios paisajes asociados, constituye un enorme yacimiento de recursos culturales de gran potencia y visibilidad y actúa como elemento estructurante de las más variadas acciones de intervención, investigación, creación y difusión de proyectos de dinamización económica y social fundamentados en el turismo.
Un tipo de proyecto muy característico son los ecomuseos. Este concepto se basa en el objetivo de convertir un territorio industrial, los diferentes elementos construidos y el paisaje cultural resultante en un museo, de manera que se establezca una relación recíproca entre la sociedad y el entorno en el que se localizaron las fábricas (Pardo Abad, 2008; Pardo Abad, 2017). Las industrias pueden mantenerse aún en activo en algunas ocasiones, pero lo más frecuente es que los ecomuseos se creen en áreas afectadas por el cierre de fábricas con el objetivo de reforzar la identidad de su pasado industrial. A diferencia de los museos tradicionales, los ecomuseos no suelen disponer de grandes colecciones de exhibición y hay que desplazarse sobre el terreno para conocer minas, fundiciones, altos hornos, casas de obreros y patronos o vías férreas. Representan un proyecto abierto y participativo que funciona de manera integrada con el territorio y muestra los distintos elementos como parte de un paisaje.

Los primeros pasos dados en esta dirección se remontan a finales del siglo XIX, en un momento de temor a que la creciente industrialización destruyera la variedad cultural de la sociedad rural. La realización más innovadora fue la apertura del museo Skansen en 1891, en Suecia, el primer museo al aire libre del mundo. En este caso se habilitó un amplio lugar para reconstruir escenas de la vida cotidiana y agrícola de Escandinavia. Este esquema museográfico se convirtió luego en un modelo a imitar en los llamados open air museums del mundo anglosajón y en los freilichtmuseen del germánico.

Otra innovación importante fue introducida gracias a los heimatmuseen. Se trataba de pequeñas instituciones museísticas creadas para poner en valor un oficio tradicional, una industria concreta o el talento de algún personaje particular. Esta experiencia conoció una difusión notable en la Alemania de entreguerras como forma de lograr una cierta cohesión social, perdida durante el conflicto de la I Guerra Mundial, y recuperar un pasado prebélico muy idealizado. La novedad del heimatmuseum consistía en colocar a la comunidad local en el centro del universo museístico, adoptando un punto de vista global en la presentación del patrimonio. El régimen nazi instrumentalizó este modelo museístico porque la focalización en la comunidad local fue entendida en términos nacionalistas, la interpretación del sentimiento de identidad territorial se proyectó de manera patriótica, la didáctica se transformó en adoctrinamiento y propaganda en torno a los valores de la raza y la dinámica propia de estos centros 
se opuso a la quietud del resto de museos. Durante el régimen nazi se crearon cerca de dos mil sitios de estas características y, más allá de su utilización política, es importante interpretarlos como síntoma de una transformación profunda de todo el panorama museístico internacional. El apogeo de los heimatmuseen fue casi contemporáneo a la creación del Museo de Artes y Tradiciones Populares de París en 1935.

Después de la II Guerra Mundial, el consumo cultural de las tradiciones populares ya no se limita a la escenificación de lo insólito y se extiende a los rasgos más cotidianos de las comunidades locales. Los museos etnográficos se orientan también hacia el mundo industrial y urbano, traspasando los límites de la cultura popular como representación idealizada del mundo rural, que tanto había caracterizado las primeras experiencias museísticas, y el traslado de construcciones auténticas o de otros elementos de gran tamaño se abandona poco a poco a favor de una revalorización in situ (Thimothy, 2011).

En la década de 1950 surgen en Estados Unidos, por influencia de los inmigrantes escandinavos, numerosos museos de pequeñas dimensiones. En los años sesenta se abre paso en Dinamarca el nuevo concepto de museo-taller, en el que al público se le invita a participar directamente, superándose la noción de los museos al aire libre de las décadas anteriores. La crisis del modelo industrial tradicional de los países desarrollados contribuyó a despertar el interés por el patrimonio industrial. En Gran Bretaña y Estados Unidos, siguiendo la referencia del tipo de museos ya comentados, se crearon muchos centros interpretativos, generalmente de reducidas dimensiones y enfocados a la historia industrial y rural de base local. Se volcaron no solo en la salvaguarda del patrimonio construi- do sino también en la conservación de las tecnologías pasadas y el saber-hacer popular.

La evolución del concepto de patrimonio cultural y los debates planteados en torno a la función de los museos permitieron la constitución de los primeros ecomuseos. Su éxito tuvo que ver con la noción de patrimonio global que se fue abriendo paso en el panorama cultural y la necesidad de una aproximación científica de tipo multidisciplinar. Las ventajas de establecer estrechos vínculos de conexión entre la comunidad y el territorio propiciaron aún más su desarrollo, lo que facilitó su proyección y vinculación casi naturales hacia el concepto cada vez más extendido de la sostenibilidad.

Las primeras experiencias surgieron tomando como referencia dos modelos diferentes: el ecomuseo ligado al medio (centrado en los valores ambientales de un lugar) y el ecomuseo comunitario (centrado en el desarrollo social de ámbito local). Los dos modelos se enmarcan en una lógica evolutiva que comprende varias fases (tabla 1):

a) La primera fase, en la primera mitad de los años setenta, se centró en el concepto de espacio.

b) En la segunda fase, cuyo comienzo se sitúa hacia 1975, se introdujeron las nociones de tiempo, territorio y comunidad.

c) La tercera fase, que se inició a principios de los años ochenta, se caracterizó por la revalorización del territorio y su patrimonio natural y cultural asociado. El crecimiento fue tan espectacular en esta fase que los ecomuseos creados entonces respondían a múltiples tipos diferentes, basados a veces en la mera agrupación espacial de recursos

Tabla 1. Fases en la evolución de los ecomuseos

\begin{tabular}{|c|c|c|c|}
\hline Fase & Periodo de inicio & Rasgo definitorio & Tipologías \\
\hline Primera fase & $\begin{array}{l}\text { Primera mitad de la década } \\
\text { de } 1970\end{array}$ & Noción de espacio & Museo de territorio \\
\hline Segunda fase & $\begin{array}{l}\text { Segunda mitad de la década } \\
\text { de } 1970\end{array}$ & $\begin{array}{l}\text { Nociones de tiempo, territorio y } \\
\text { comunidad }\end{array}$ & Museo de territorio \\
\hline Tercera fase & $\begin{array}{l}\text { Comienzos de la década de } \\
1980\end{array}$ & $\begin{array}{l}\text { Revalorización del patrimonio na- } \\
\text { tural y cultural }\end{array}$ & $\begin{array}{l}\text { Ecomuseo de micro-historia } \\
\text { Museo de lugar } \\
\text { Pueblo-museo } \\
\text { Ecomuseo en red }\end{array}$ \\
\hline Cuarta fase & Finales de la década de 1990 & $\begin{array}{l}\text { Refuerzo de las identidades locales } \\
\text { y desarrollo territorial }\end{array}$ & Todas las anteriores \\
\hline
\end{tabular}

Fuente: elaboración propia. 
para una explotación turística lo más intensiva posible y alejada de los principios más elementales de la sostenibilidad.

d) La cuarta fase surge a finales de la década de 1990. Las motivaciones de carácter económico persisten y se añaden, como un nuevo estímulo, las preocupaciones ligadas a la globalización y la uniformidad de las identidades locales. El papel de los poderes públicos aumenta en estos momentos y, por el contrario, disminuyen las iniciativas privadas. Muchos de los ecomuseos creados ahora se enmarcan en las políticas económicas y sociales de ámbito local y regional, orientadas al fomento del empleo, el uso sostenible de los recursos culturales y naturales, el fomento de la identidad comunitaria y la participación activa de la población y los agentes del lugar.

\section{RETOS PARA UN TURISMO SOSTENIBLE DE PATRI- MONIO INDUSTRIAL: EL VALOR DEL PAISAJE}

Las actividades productivas y extractivas han dejado a lo largo del tiempo unas huellas de gran alcance sobre el territorio. Son las muestras de un proceso pertinaz de conformación de paisajes, con unos rasgos comunes en muchos casos que no impiden su distinción única.

La difusión de técnicas, a través de los intercambios establecidos entre países y regiones, permitió crear unos modelos parecidos de explotación de los recursos naturales y de producción. Esto facilita en la actualidad que muchos territorios de la industrialización compartan experiencias de difusión de los valores asociados al patrimonio industrial, propuestas de buenas prácticas para el desarrollo sostenible, asesoramiento en la mejora del medio ambiente o introducción de nuevos usos turísticos. El encuentro de puntos comunes entre distintos lugares vinculados inicialmente a la industria no impide enfatizar las características diferenciadoras de cada sitio en particular.

Las experiencias compartidas garantizan un mejor futuro para el turismo sostenible de patrimonio industrial. En muchos proyectos ya consolidados, tanto de España como de otros países europeos, ese futuro es ya una realidad y la recuperación de los vestigios materiales se ha convertido en un motor de desarrollo económico en muchas comarcas inicialmente marcadas por el cierre y el abandono de las explotaciones ${ }^{3}$. La experiencia acumulada en esos proyectos demuestra la necesidad de contemplar siempre una serie de estrategias de intervención para alcanzar el objetivo irrenunciable del desarrollo sostenible (figura 2): a) La ordenación del territorio. El patrimonio de la industrialización ha sido un eje vertebrador y dinamizador de numerosos lugares. Tanto el patrimonio industrial como el paisaje cultural que genera deben de incorporarse como elementos fundamentales de la planificación del territorio, de las políticas urbanísticas aplicadas o de tipo cultural, medioambiental, social y económico.

b) La concepción de estos territorios como paisajes culturales. Para ello es imprescindible implementar las medidas específicas que se establecen al respecto en el Convenio Europeo del Paisaje, combinando de manera ordenada la intervención sobre los distintos elementos heredados con la preservación del paisaje y la recuperación ambiental de las zonas degradadas.

c) La propiedad de los vestigios. La titularidad es un factor determinante en el desarrollo turístico de los enclaves y para ello se ha de hacer cumplir la legislación sobre protección de bienes culturales cuando son de propiedad privada. En otras ocasiones puede ser interesante propiciar el paso a titularidad pública de los elementos patrimoniales más significativos, así como concienciar de su valor para la generación de riqueza y empleo.

d) La implicación de las administraciones locales y agentes sociales. Este aspecto es particularmente importante para el éxito final de las iniciativas de nuevo uso, ya que pueden propiciar y garantizar la participación activa de la comunidad. Las actividades dirigidas a conocer y difundir los valores del patrimonio industrial entre la población local son muy importantes, así como la formación de personal cualificado para la correcta gestión del patrimonio.

e) El desarrollo de un turismo sostenible. El nuevo uso turístico es una vía activa de desarrollo socioeconómico. Es imprescindible poner en valor los recursos disponibles mediante proyectos creativos e imaginativos, de forma que realcen los valores particulares. Lo más distintivo y diferenciador de cada lugar debe servir para crear productos turísticos de calidad que promuevan el patrimonio material e inmaterial y la identidad local con museos especializados, rutas ecoculturales, visitas a minas, instalaciones industriales, centros de interpretación interactivos, etc.

Más allá de las estrategias de intervención que se acaban de analizar, un aspecto muy importante que hay que considerar es la sostenibilidad económica de los proyectos. La limitada capacidad de atracción de 
los espacios mineros o industriales musealizados convierte en más vulnerables las iniciativas de nuevo uso turístico y cultural. Los gastos iniciales son cuantiosos por la envergadura de las estructuras construidas y la amplitud de los lugares afectados, a lo que se une el hecho indiscutible de que los beneficios, cuando existen, son reducidos frente a otras opciones de turismo (Prat Forga y Díaz Soria, 2013).

La sostenibilidad económica es bastante discutible en la mayor parte de los casos, debido básicamente a que el turismo en el entorno del legado de la industrialización es minoritario. Ello obliga a que sea imprescindible una gestión eficaz de los recursos económicos disponibles, el apoyo financiero de las administraciones públicas y el patrocinio de algunas entidades privadas para lograr suplir unos ingresos propios insuficientes. Solo así se garantiza la continuidad de las propuestas y la consideración turística de unos destinos muchas veces alejados de los principales itinerarios culturales. El turismo, tal y como se reconoce en la Carta Mundial del Turismo Sostenible +20, aprobada en el año 2015, es una fuerza motriz del patrimonio cultural capaz de dinamizar la economía de las comunidades locales si se adoptan, en línea con los Objetivos del Desarrollo
Sostenible (ODS) de las Naciones Unidas, modelos de gestión innovadores y apropiados para mejorar la eficiencia del uso de los recursos ${ }^{4}$.

Esta misma línea argumental aparece recogida en la ya comentada revisión del Plan Nacional de Patrimonio Industrial del año 2011, afirmando que la conservación y activación del patrimonio cultural nunca han sido ajenas a la situación del entorno económico ni a los cambios de paradigma en la intervención patrimonial. El énfasis de la sostenibilidad obliga necesariamente a elegir determinadas alternativas frente a la priorización tradicional de inversión de los recursos, tanto públicos como privados. La generación de una nueva Cultura del Patrimonio supone que la sociedad y las instituciones se han de implicar en el reconocimiento de los valores asociados al legado de la industrialización, pero también que se extienda la gestión proactiva de los recursos patrimoniales, el disfrute de los mismos por parte de la población y el fomento de los programas de desarrollo sostenible de escala local y regional ${ }^{5}$.

A pesar de la superación de muchos de los retos planteados, la situación actual del patrimonio industrial dista mucho de estar completamente resuelta, como consecuencia de la presencia pertinaz de gra-

Figura 2. Estrategias de intervención para un turismo sostenible de patrimonio industrial

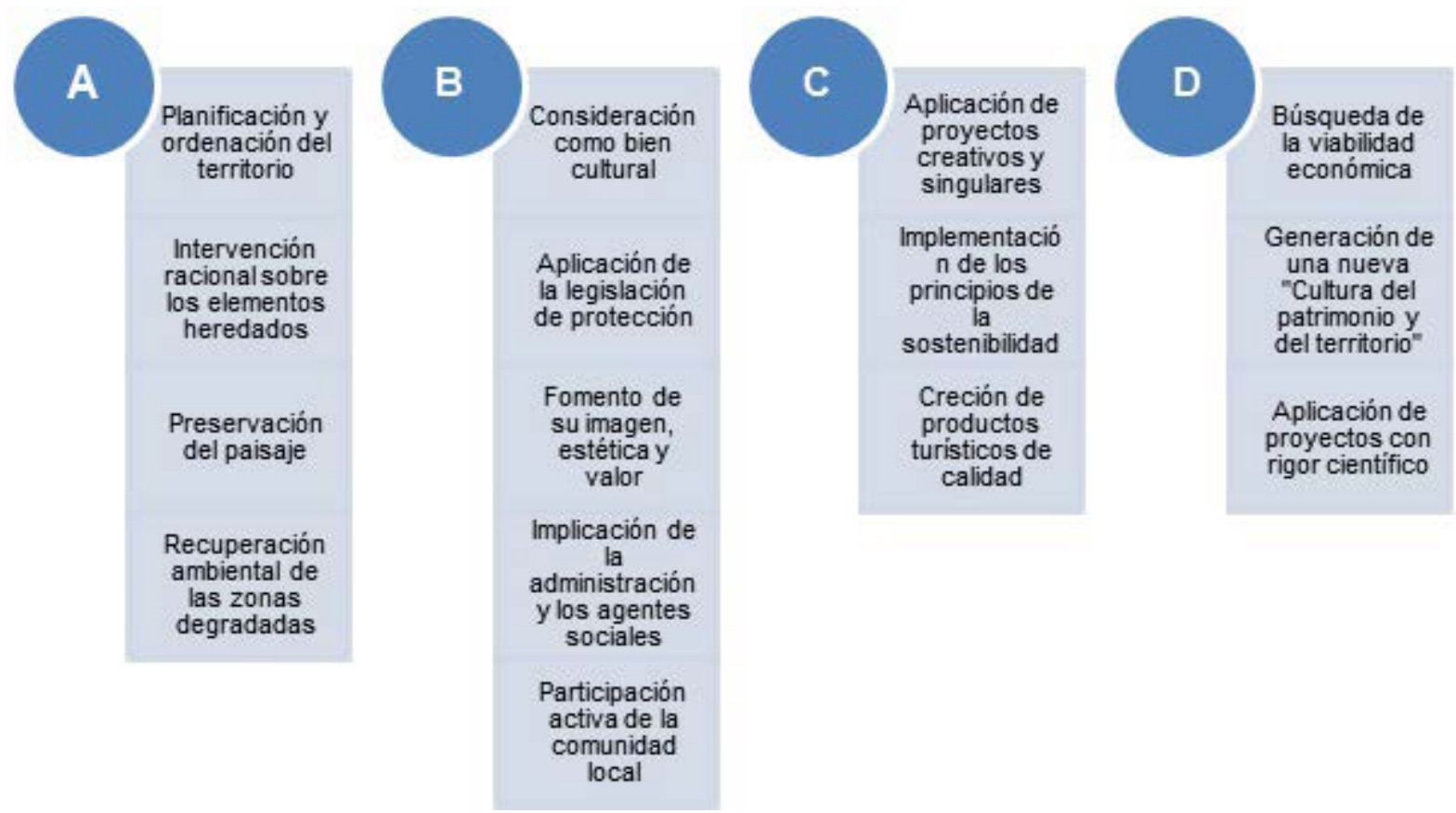

\footnotetext{
A. Interpretación de los territorios antes industriales como paisajes culturales

B. Protección del patrimonio minero-industrial.

C. Fomento del turismo.

D. Gestión eficaz y proactiva de los recursos disponibles.
} 
ves amenazas que se ciernen sobre un legado muy específico, a la vez que muy amplio e insuficientemente reconocido.

Es un hecho constatado que el uso turístico del patrimonio industrial no siempre responde a las exigencias de la sostenibilidad ni del desarrollo responsable. El gran aporte que representa la actividad turística para la revitalización de pueblos, comarcas y ciudades, antes alejadas de los centros más dinámicos e innovadores, ha ido en paralelo a un cambio en las prácticas de los consumos culturales. Este patrimonio ha sido objeto de atención como un nuevo recurso puesto al servicio de la renovación de las identidades en las áreas desindustrializadas, valorándose como un hito o icono frente a la uniformidad global. En este cambio de apreciación no se han aplicado siempre las debidas estrategias de desarrollo sostenible, lo que retrasa la plena recuperación de unos territorios bastante vulnerables.

El cambio en los consumos culturales ha incentivado un aumento constante de los proyectos de intervención sobre los elementos patrimoniales, procurando satisfacer la demanda de experiencias diferenciadas de los modelos tradicionales. Esto reduce la posibilidad de implementar proyectos con mayor rigor científico, tanto en bienes pequeños y aislados como en grandes conjuntos industriales o mineros de gran alcance territorial.

Otra amenaza importante es que se ha considerado el patrimonio industrial casi exclusivamente desde una perspectiva arquitectónica centrada en los bienes inmuebles. Se han obviado con gran frecuencia los bienes muebles y el patrimonio intangible vinculado a tradiciones, conocimientos, creencias o eventos de la industrialización. No se ha apostado por el mantenimiento global de un legado muy extenso, pero fundamental en todas sus facetas para comprender la historia técnica, económica y social de los territorios. La pérdida de patrimonio mueble e intangible ha sido permanente y ha acompañado al proceso constante de abandono y cierre de fábricas, trabajos, oficios y deslocalización de empresas y personas en los lugares de antigua industrialización. El de tipo inmueble, aunque mejor considerado, se ha visto afectado por un proceso de ocupación o sustitución por otros usos (residenciales y terciarios, por ejemplo), que han motivado importantes desapariciones de elementos. Esto ha sido particularmente intenso en las ciudades, al amparo de tensiones de corte urbanístico y especulativo que han materializado un cambio radical del paisaje en muchos espacios fabriles de carácter histórico.
Todas estas amenazas se han consumado con demasiada frecuencia ante las deficiencias existentes en la protección y conservación de un patrimonio poco valorado. La ausencia de figuras normativas y legales, de una planificación integral de los recursos heredados de la industrialización, de una mayor coordinación entre las diferentes administraciones públicas, y entre estas y el sector privado, y el particular reparto de competencias en materia de patrimonio en el caso español son otras amenazas añadidas a una situación de permanente vulnerabilidad.

\section{DISCUSIÓN EN TORNO AL MÉTODO DE ANÁLISIS DE ALGUNAS EXPERIENCIAS TURÍSTICAS}

Los paisajes industriales y los valores escénicos asociados forman parte de nuevos tipos de turismo. Su poder de información y su capacidad para transmitir conocimientos superan, en muchas ocasiones, los más especializados museos. Además, suelen estar presentes dos elementos interpretativos fundamentales: el territorio y el medio ambiente. No hay que olvidar que la concienciación medioambiental en los países occidentales, iniciada a finales de la década de 1960 y continuada en la de 1970, coincide en el tiempo con el creciente interés por la preservación de los monumentos industriales (Tempel, 2012).

Las labores de recuperación ambiental y de descontaminación de suelos han sido, de hecho, muy importantes en algunos enclaves concretos. Más, incluso, que la propia preservación del patrimonio construido. Es la forma más habitual de proyectar modelos sostenibles y disimular los efectos acumulados derivados de los usos y abusos producidos. El paisaje de Riotinto, en la provincia de Huelva, es un ejemplo espectacular por ser testimonio de la gran magnitud de las labores mineras a cielo abierto (figura 3). Es muy impactante para cualquier observador, y tal vez se trate de una de las zonas postindustriales con mayores valores escénicos de todo el continente por la gran variedad cromática de las tierras y restos minerales. Esa es su principal fortaleza como destino de visitantes. La renaturalización del paisaje no se ha considerado necesaria, al contrario de las operaciones emprendidas en algunas cuencas hulleras y áreas industriales altamente contaminadas (brownfields to greenfields).

La zona de Almadén, en la provincia de Ciudad Real, es diferente por tratarse de una minería subterránea de menor escala, aunque los valores arquitectónicos y patrimoniales fueron reconocidos el 1 de julio de 2012 como Patrimonio de la Humanidad. En ambos casos, los restos son perfectamente accesibles para 
Figura 3. Zona minera de Riotinto (Huelva)

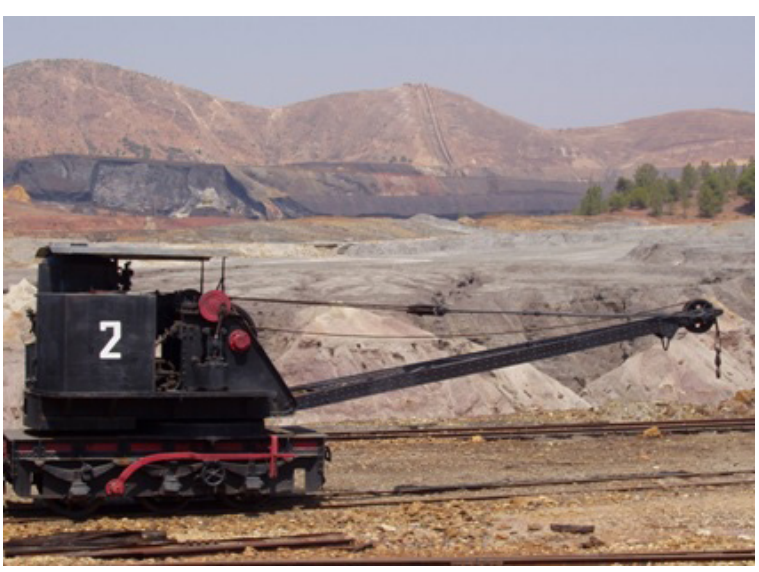

Fotografía tomada en agosto de 2006.

el público, facilitando una interpretación de conjunto que abarca también las condiciones laborales y familiares de los mineros. Este doble aspecto estimula las visitas, en este y en otros enclaves de patrimonio industrial (Shackel y Palus, 2006).

En ambos lugares se ha optado por la creación de sendos parques mineros como mejor solución de cara a su nuevo uso turístico. No solo porque cada uno de ellos encierra un área homogénea, sino porque representan una estrategia de conjunto muy interesante que integra la preservación del patrimonio construido y el ambiente geológico en el que se produjo la actividad minera. Las poblaciones locales han acogido muy bien las dos experiencias de reutilización porque las entienden como una forma de continuidad con el pasado y de generación de empleo y riqueza.

Las dificultades iniciales se han superado por la extraordinaria coordinación de todos los agentes implicados. Ha sido clave el papel desempeñado por las autoridades municipales, la población local y el impulso ofrecido por las compañías mineras. Esto demuestra que es imprescindible la intervención coordinada de agentes, no solo la calidad de los proyectos, en la recuperación turística del legado industrial y en la superación de cualquier dificultad.

El estudio concreto de las relaciones entre turismo de patrimonio industrial y la sostenibilidad, en estos dos enclaves citados y en otros más, forma parte de una de nuestras líneas específicas de investigación, con resultados previos publicados en varias revistas científicas (Pardo Abad, 2014; Pardo Abad, 2017). Son aproximaciones en las que se aborda un estudio basado en la combinación de enfoques metodológicos cualitativos y cuantitativos y en la revisión crítica de las teorías vinculadas a la sostenibilidad. Han sido dos aportaciones temáticas novedosas, hasta ahora no analizadas en profundidad, que se fundamentan en la definición de conceptos claves y en el cumplimiento de una serie de indicadores de significación turística, cultural, económica, social y ambiental.

Se crearon en ambos casos un número significativo de indicadores específicos, agrupados en varios componentes diferentes, y se diseñó un índice sintético de desarrollo sostenible (ISDS), calculado como media para los valores obtenidos en los indicadores seleccionados. Los componentes usados son los siguientes (tabla 2): gestión integrada de los recursos naturales y culturales, generación de beneficios sociales y económicos, generación de beneficios culturales y generación de beneficios ambientales. Cada uno de esos componentes se desglosó en un número variable de indicadores relacionados. Un indicador es una herramienta que permite la obtención de información necesaria para comprender los vínculos y los impactos, en este caso, del turismo con respecto al entorno industrial o minero en el que se desenvuelve. Partiendo de esta consideración, se puede afirmar que sirven para medir, comparar y facilitar la toma de decisiones referidas a la gestión y ordenación de los recursos. Es muy importante que dichos indicadores cumplan necesariamente con una serie de criterios que se consideran imprescindibles: validez científica, representatividad, relevancia, comparabilidad, cuantificación, cobertura geográfica y fiabilidad. De esta forma se consigue una medida de sostenibilidad de tipo multidimensional, fácilmente comprensible, permitiendo el análisis comparativo entre las diferentes áreas seleccionadas para su investigación.

Se efectuaron también entrevistas a los gestores de los enclaves turísticos seleccionados de patrimonio industrial y a los responsables políticos y técnicos de los municipios respectivos. Con la intención de valorar las respuestas en relación a la sostenibilidad turística final, se estableció un control de aplicación directa entre 0 y 4 en cada una de las respuestas. De esta manera fue posible establecer los cálculos necesarios para conocer el valor medio de cada componente y el índice sintético de desarrollo sostenible final en cada lugar, entendido como media del conjunto de los indicadores utilizados. Las directrices generales recogidas por algunas instituciones públicas para la cuantificación de indicadores, como la Oficina Europea de Estadística (EUROSTAT), la Agencia Europea del Medio Ambiente, el Ministerio de Medio Ambiente o el Instituto Nacional de Estadística (INE), fueron un referente fundamental en esta parte de la investigación. 
Tabla 2. Componentes e indicadores de sostenibilidad turística de patrimonio industrial

\begin{tabular}{|c|c|}
\hline $\begin{array}{l}\text { Componentes de gestión } \\
\text { (CG) }\end{array}$ & $\begin{array}{l}\text { I Nivel de protección patrimonial } \\
I_{2} \text { Nivel de rehabilitación patrimonial } \\
I_{3} \text { Cumplimiento de la zonificación local } \\
I_{4} \text { Información proporcionada a los visitantes } \\
I_{5} \text { Dotación de fondos económicos } \\
I_{6} \text { Aportación económica de las entidades locales }\end{array}$ \\
\hline $\begin{array}{l}\text { Componentes sociales y económicos } \\
\text { (CSE) }\end{array}$ & $\begin{array}{l}I_{7} \text { Beneficios para la comunidad local } \\
I_{8} \text { Colaboración de la población local } \\
I_{9} \text { Mejora de las infraestructuras } \\
I_{10} \text { Incremento de los equipamientos } \\
I_{11} \text { Generación de nuevo empleo } \\
I_{12} \text { Procedencia local de los trabajadores empleados }\end{array}$ \\
\hline $\begin{array}{l}\text { Componentes culturales } \\
\text { (CC) }\end{array}$ & $\begin{array}{l}I_{13} \text { Nivel de satisfacción general de las visitas } \\
I_{14} \text { Impulso de la cultura local } \\
I_{15} \text { Dinamización de la oferta cultural local } \\
I_{16} \text { Realización de actividades paralelas externas } \\
I_{17} \text { Nivel de satisfacción de la comunidad } \\
I_{18} \text { Apreciación de la población hacia el patrimonio industrial }\end{array}$ \\
\hline $\begin{array}{l}\text { Componentes ambientales } \\
\text { (CA) }\end{array}$ & $\begin{array}{l}I_{19} \text { Nivel de recuperación ambiental del entorno } \\
I_{20} \text { Consideración estética del entorno recuperado } \\
I_{21} \text { Uso de fuentes energéticas renovables } \\
I_{22} \text { Aplicación de medidas de ahorro energético } \\
I_{23} \text { Conexión del patrimonio construido con el entorno natural }\end{array}$ \\
\hline
\end{tabular}

Fuente: Pardo Abad (2014).

Para analizar la sostenibilidad del turismo en los lugares considerados se agregó la información en dos fases. En la primera se obtuvo un indicador sintético para cada componente (de gestión, social, económico, cultural y medioambiental). En la segunda fase se calculó un indicador sintético global que proporcionase una medida de carácter multidimensional de turismo sostenible. El análisis final de los valores obtenidos permitió determinar las características de cada destino, estudiando la situación relativa de cada componente e indicador dentro del conjunto. Este método de análisis permite la comparación entre las zonas seleccionadas, identificando semejanzas y diferencias en cuanto al nivel de sostenibilidad: conservación del patrimonio cultural, dotación de infraestructuras, provisión de equipamientos, grado de información turística, creación de empleo, promoción y revitalización de la cultura local, implementación de medidas de eficiencia energética, conexión del patrimonio construido con el medio natural, etc.

En los dos estudios se pudieron alcanzar algunas conclusiones relevantes, como el hecho de que la sostenibilidad está ligada sobre todo a proyectos a medio y largo plazo. También se concluyó que, a mayor nivel de sostenibilidad, mayor número de visitas turísticas, es decir, el uso y la gestión sostenible de los lugares y paisajes de patrimonio industrial se acaba convirtiendo en un importante reclamo para el turismo, lo que, por otra parte, confirma el hecho argumentado desde numerosos trabajos de que el turismo, hábilmente utilizado, es una condición para la conservación del patrimonio. Evidentemente, la frecuentación turística, siempre que se asegure desde criterios de sostenibilidad, genera nuevas expectativas de empleo e incrementa el desarrollo de las comunidades locales. Los dos trabajos referidos han permitido crear un modelo de valoración de ámbito local en enclaves concretos, pero con aspiraciones a convertirse en un referente analítico en otros paisajes culturales asociados a la industrialización.

\section{CONCLUSIONES}

Los proyectos de recuperación y puesta en valor emprendidos en los antiguos enclaves mineros e industriales tienen en la actualidad un alcance turístico de gran interés por sus implicaciones de carácter medioambiental, territorial y patrimonial: han logrado poner en valor numerosos elementos heredados, atraer un 
significativo flujo de visitantes, estimular el turismo y el sector servicios en áreas de interior alejadas tradicionalmente de los circuitos turísticos y recuperar, bajo criterios sostenibles, el territorio dedicado durante tanto tiempo a las labores extractivas o productivas.

La recuperación de la memoria colectiva ha resultado ser también un aspecto fundamental, lo que da coherencia a las intervenciones y asegura su continuidad. Algunos de los mejores ejemplos al respecto, como Riotinto y Almadén, han aplicado programas de protección patrimonial de gran interés e impacto por su carácter integral y los resultados alcanzados en relación con la sostenibilidad y el uso turístico.

En la recuperación deben jugar un papel decisivo los ayuntamientos y otras entidades locales, de forma que permitan la coordinación de todos los agentes que participan de la intervención. Esto facilita el éxito final de los nuevos proyectos y demuestra la necesidad de lograr los máximos niveles de sinergia en la recuperación del patrimonio industrial. Otro factor de éxito indiscutible es que las empresas mineras o industriales, responsables de cada explotación, se im- pliquen en los proyectos, así como la participación de los antiguos trabajadores y la población local. Solo de esta forma se integrará la experiencia como un activo fundamental para incentivar las visitas y hacer comprensible la interpretación del lugar, su paisaje y los bienes heredados.

El paisaje es un factor de atracción de primera magnitud, ya que los valores estéticos y visuales asociados a los territorios de vieja industrialización son muy elevados. La espectacularidad de los sitios de producción y extracción, los contrastes cromáticos de los suelos, las técnicas y maquinarias empleadas para el movimiento de tierras o las infraestructuras vinculadas crean unos entornos extraordinarios y muy singulares con gran poder de fascinación. Evocan fácilmente otras épocas y el enorme esfuerzo aplicado para obtener de la naturaleza los recursos necesarios para el progreso de la sociedad. Todo esto constituye la seña de identidad de los nuevos productos turísticos ofertados en el entorno del patrimonio industrial, con base en un desarrollo sostenible que garantiza el éxito actual de los proyectos y su permanencia futura.

\section{NOTAS}

1. Así lo indicó Manuel Antonio Zárate en "Les paysages culturels espagnols. Le besoin de conciliation entre l'amenegement et le patrimoine", contribución inédita al XXXI Congrès International de Géographie de la UGI (Túnez, 2008).

2. Desde 1986 figura en la lista del Patrimonio Mundial de la UNESCO y desde 2009 recibe más 450.000 visitantes al año.

3. Así lo indicó A. A. Pérez en "Recuperación del patrimonio minero y desarrollo sostenible", contribución presentada a las VII Jornadas sobre alternativas de desarrollo en comarcas mineras (La Unión, 2008).

\section{BIBLIOGRAFÍA}

Albrecht, H. (2012). What does the Industrial Revolution signify? En Douet, J. (ed.). Industrial Heritage Re-tooled. The TICCIH Guide to Industrial Heritage Conservation. Lancaster: Carnegie Publishing Ltd. / The International Committee for the Conservation of the Industrial Heritage (TICCIH), pp. 17-23.

Álvarez, M. A. (ed.) (2010). Patrimonio industrial y paisaje. Actas del V Congreso para la Conservación del Patrimonio Industrial y de la Obra Pública en España. Gijón: TICCIH-España.
4. La idea de preservar el patrimonio común es clave en la Carta Mundial del Turismo Sostenible +20 . En ella se afirma lo siguiente: "El patrimonio es uno de los motores principales del turismo, y el turismo sostenible debe ser entendido como el motor de desarrollo de los sitios patrimonio. La conservación, la protección y la puesta en valor, de forma inteligente y responsable, del patrimonio natural y cultural, representa por lo tanto un ámbito privilegiado para la cooperación y la innovación". En el concepto de patrimonio se incluye también lo inmaterial, transmitido de generación en generación y que infunde a las comunidades un sentimiento de identidad y continuidad. Esto aporta a los destinos, como valores reconocidos, la autenticidad y la integridad.

5. El éxito de la preservación del patrimonio industrial, tal y como se expresa en el citado Plan Nacional de Patrimonio Industrial (2011), depende en gran medida de las posibilidades de contribuir al desarrollo local, regenerando lugares y potenciando el turismo.
Benito, P. (2012). Territorio, paisaje y herencia industrial: debates y acciones en el contexto europeo. Documents d'Anàlisi Geogràfica, 58 (3), pp. 443-457. https:// doi.org/10.5565/rev/dag.11

Benito, P. y Alonso, P. (2012). Industrial Heritage and Place Identity in Spain: from Monuments to Landscapes. The Geographical Review, 102 (4), pp. 446-464. https://doi. org/10.1111/j.1931-0846.2012.00169.x

Benko, G. y Strohmayer, U. (eds.) (1997). Space and Social Theory: Interpreting
Modernity and Postmodernity. Blackwell Publishers.

Butler, R. (1999). Sustainable tourism: a state-of-the-art review. Tourism Geographies, 1 (1), pp. 7-25. https://doi. org/10.1080/14616689908721291

Casanelles, E. (1998). Recuperación y uso del patrimonio industrial. Ábaco. Revista de Cultura y Ciencias Sociales, 19, pp. 11-18.

Castillo, A. M., López, T. J. y Millán, G. (2010). El turismo industrial minero 
como motor de desarrollo en áreas geográficas en declive. Un estudio de caso. Estudios y Perspectivas en Turismo, 19 (3), pp. 382-393.

Coccossis, H. (1996). Tourism and sustainability: perspectives and implications. En Priestley, G. K., Edwards, J. A. y Coccossis, H. (eds.). Sustainable tourism? European experiences. Oxford: CAB International, pp. 1-21.

Cole, D. (2004). Exploring the sustainability of mining heritage tourism. Journal of Sustainable Tourism, 12 (6), pp. 480-494. https://doi. org/10.1080/09669580408667250

Cossons, N. (2012). Why preserve the Industrial Heritage? En Douet, J. (ed.). Industrial Heritage Re-tooled. The $\mathrm{TICCIH}$ guide to Industrial Heritage Conservation. Lancaster: Carnegie Publishing Ltd. / The International Committee for the Conservation of the Industrial Heritage (TICCIH), pp. 6-16.

Dambron, P. (2004). Patrimoine industriel et développement local. Paris: Éditions Jean Delaville.

Edwards, A. y Llurdés, J. A. (1996). Mines and quarries. Annals of Tourism Research, 23 (2), pp. 341-362. https://doi. org/10.1016/0160-7383(95)00067-4

EUROSTAT (2006). Estrategia de Desarrollo Sostenible de la Unión Europea. Bruselas: Oficina Europea de Estadística.

Feliú Torras, A. (1998). El patrimonio industrial, localizaciones, regeneraciones: una nueva geografía. Ábaco. Revista de Cultura y Ciencias Sociales, 19, pp. 71-80.

Hidalgo, C. (2010). El patrimonio mineroindustrial y ferroviario: nuevos recursos para nuevos turismos. Madrid: Universidad Autónoma de Madrid.

Hospers, G.-J. (2002). Industrial heritage tourism and regional restructuring in the European Union. European Planning Studies, 10, pp. 397-404. https:// doi.org/10.1080/09654310220121112

Jansen-Verbeke, M. (1999). Industrial Heritage: A Nexus for Sustainable Tourism Development. Tourism Geographies, 1 (1), pp. 70-85. https://doi. org/10.1080/14616689908721295

Jones, C. y Munday, M. (2001). Blaenavon and United Nations World Heritage Site Status: Is Conservation of Industrial Heritage a Road to Local Economic Development? Regional Studies, 35, pp. 585-590. https:// doi.org/10.1080/00343400120065741
Ortega Cantero, N. (2010). El lugar del paisaje en la geografía moderna. Estudios Geográficos, 71 (269), pp. 367393. https://doi.org/10.3989/estgeogr.201012

Pardo Abad, C. J. (1990). Cambios de uso de suelo en la ciudad: vaciado industrial y renovación urbana en Madrid. [Tesis doctoral inédita]. Universidad Autónoma de Madrid: Madrid.

Pardo Abad, C. J. (2008). Turismo y patrimonio industrial. Un análisis desde la perspectiva territorial. Madrid: Síntesis.

Pardo Abad, C. J. (2010). El patrimonio industrial en España: análisis turístico y significado territorial de algunos proyectos de recuperación. Boletín de la Asociación de Geógrafos Españoles, 53, pp. 239-266.

Pardo Abad, C. J. (2014). Indicadores de sostenibilidad turística aplicados al patrimonio industrial y minero: evaluación de resultados en algunos casos de estudio. Boletín de la Asociación de Geógrafos Españoles, 65, pp. 11-36.

Pardo Abad, C. J. (2016). El patrimonio industrial en España. Paisajes, lugares y elementos singulares. Madrid: Akal.

Pardo Abad, C. J. (2017). The post-industrial landscapes of Riotinto and Almadén, Spain: scenic value, heritage and sustainable tourism. Journal of Heritage Tourism, 12 (4), pp. 331-346. https://doi.or g/10.1080/1743873X.2016.1187149

Pérez, L. y Parra, C. (2004). Paisajes culturales: el parque patrimonial como instrumento de revalorización y revitalización del territorio. Teoría, 13, pp. 9-24.

Prat Forga, J. M. y Díaz Soria, I. (2013). Análisis de la sostenibilidad económica del turismo industrial. El caso de Cataluña. TURyDES, 6 (14), pp. 1-13. Disponible en: http://www.eumed.net/rev/turydes/14/ turismo-industrial-cataluna.pdf

Sabaté Bel, J. (2004). ¿Paisajes culturales, consecuencia de la postmodernidad? II Seminari Internacional sobre Paisatge. Barcelona: Consorci Universitat Internacional Menéndez Pelayo de Barcelona, pp. 1-19. Disponible en: http://www.coac.net/ofpaisatge/content/2004/2004_joaquin_sabate.pdf

Samuel, R. (1994). Theatres of Memory: Past and Present in Contemporary Culture. Londres: Verso.

Shackel, P. A. y Palus, M. (2006). Remembering an Industrial Landscape. International Journal of Historical Archaeology, 10
(1), pp. 49-71. https://doi.org/10.1007/ s10761-006-0004-4

Sharpley, R. (2000). Tourism and Sustainable Development: Exploring the Theoretical Divide. Journal of Sustainable Tourism, 8 (1), pp. 1-19. https://doi. org/10.1080/09669580008667346

Stuart, I. (2012). Identifying industrial landscapes. En Douet, J. (ed.). Industrial Heritage Re-Tooled: The TICCIH Guide to Industrial Heritage Conservation. Lancaster: Carnegie Publishing / The International Committee for the Conservation of the Industrial Heritage ( $\mathrm{TICCIH})$, pp. 48-54.

Tempel, N. (2012). Post-industrial landscapes. En Douet, J. (ed.). Industrial Heritage Re-tooled. The TICCIH guide to Industrial Heritage Conservation. Lancaster: Carnegie Publishing / The International Committee for the Conservation of the Industrial Heritage (TICCIH), pp. 142-148.

Thimothy, D. J. (2011). Cultural Heritage and Tourism. An Introduction. Bristol: Channel View Publications.

Vahí, A. (2010). Patrimonio industrial como recurso para un turismo sostenible: la cuenca del Guadalfeo (Granada). Cuadernos Geográficos, 46, pp. 65-91.

Valenzuela, M., Palacios, A. J. e Hidalgo, C. (2008). La valorización turística del patrimonio minero en entornos rurales desfavorecidos. Actores y experiencias. Cuadernos de Turismo, 22, pp. 231-260.

Zárate Martín, M. A. (2011). La visita de empresa, otra forma de hacer turismo. Estudios Geográficos, LXXII (270), pp. 291-321. https://doi.org/10.3989/estgeogr.201112

Zárate Martín, M. A. (2012). Geografía urbana. Dinámicas locales, procesos globales. Madrid: Universidad Nacional de Educación a Distancia.

\section{Otros recursos}

Desarrollo sostenible 2008. Principales indicadores de España para el seguimiento de la Estrategia de DS de la UE. Madrid: Instituto Nacional de Estadística, 2009. Disponible en http://goo.gl/L2pt7w

Plan Nacional de Patrimonio Industrial (2011). Madrid: Ministerio de Educación, Cultura y Deporte, 2011. Disponible en: http://www.mecd.gob.es/ planes-nacionales/planes-nacionales/ patrimonio-industrial.html 